\title{
SPONTANEOUS, UNREMITTING GROSS HEMATURIA OCCURRING ONE WEEK AFTER LAPAROSCOPIC DONOR NEPHRECTOMY
}

\author{
STEVE Y. CHUNG, CHRIS H. CHON, CHRISTOPHER S. NG, \\ GERHARD J. FUCHS \\ Cedars-Sinai Medical Center, Endourology Institute, Los Angeles, California, USA
}

\begin{abstract}
Complications associated with the ureteral stump after nephrectomies rarely occur, especially after donor nephrectomies. The potential for the slippage of clips is a well-known event associated with vascular ligations. We report on the first case of clip slippage from the ureter and describe diagnosis and management of the most extreme of morbid presentations.
\end{abstract}

Key words: kidney; laparoscopy; organ donation; nephrectomy; ureter; hematuria Int Braz J Urol. 2004; 30: 398-9

\section{INTRODUCTION}

The laparoscopic donor nephrectomy (LDN) is emerging as a standard for living kidney procurement. Decreased donor morbidity has been well documented with no prior reports of ureteral complications. We report a case of spontaneous, unremitting gross hematuria from the ureteral stump occurring during convalescence after an uncomplicated LDN. Diagnostic and management issues are discussed.

\section{CASE REPORT}

A healthy 23-year-old woman was discharged 2 days after an uneventful hand-assisted left LDN with ligation of the ureter using 2 titanium clips (Figure$1, \mathrm{~A})$. One week later, she developed sudden onset gross hematuria and suprapubic pain. In the emergency department, a hematuria catheter was inserted and irrigated for many clots. Her hematocrit was stable at $37 \%$ and urine culture was negative.

During 23-hour observation with catheter drainage, she had further episodes of gross hematuria requiring manual irrigation. She was subsequently taken to the operating room, and cystoscopy revealed blood clots in the left ureteral orifice. Semi-rigid ureteroscopy revealed bleeding without any identifiable source, and retrograde injection of contrast showed extravasation into the peritoneum (Figure-2). With a hematocrit of $27 \%$, she was immediately sent for angiogram, yet no active bleeder could be identified.

With persistent hematuria, the patient underwent a diagnostic exploratory laparoscopy. The approach utilized the previous umbilical and lateral port sites and one new port site. During inspection, it was evident that the titanium ureteral clips had fallen off, but no hematoma, urinoma, or active intraabdominal bleeding could be identified. The ureteral stump was eventually mobilized off the iliac vessels, and the proximal end was ligated with 2 Hem-o-lok clips (Weck Closure Systems, Research Triangle Park, NC) and wrapped with Surgicel (Figure-1, B). A Foley catheter was left in place.

By postoperative day 1, the suprapubic pain had resolved, and Foley catheter was removed. She was discharged the following day with clear, spontaneous urine output. At one-month follow-up, she denied any further episodes of gross hematuria. 


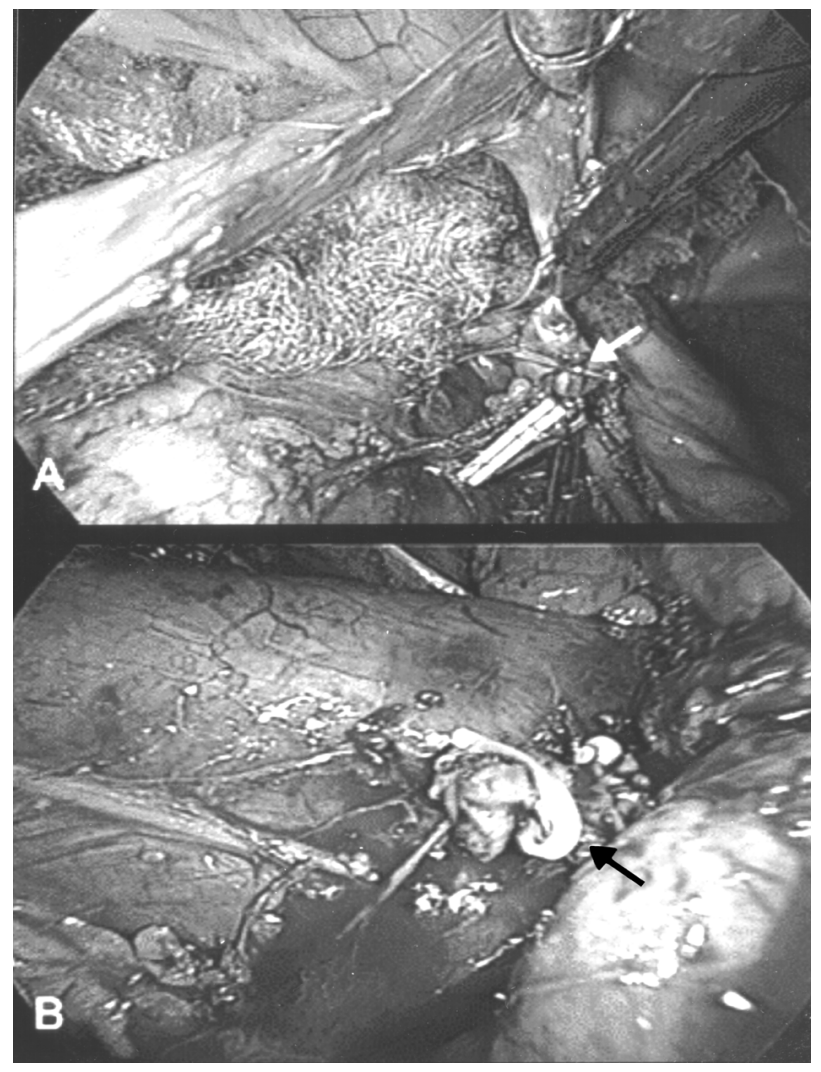

Figure 1 - A) Left ureter mobilized and excised after ligating with titanium clips during laparoscopic donor nephrectomy (arrow). B) Locking clips applied to left ureteral stump during exploratory laparoscopy.

\section{COMMENTS}

Morbidity associated with the ureteral stump after LDN is extremely rare. In a series of 738 consecutive living donor nephrectomies, Jacobs et al. reported no major or minor postoperative complications associated with the donors' ureteral stumps (1).

Based on the intraoperative findings of this case, we concede that the titanium clips on the ureteral stump suddenly dislodged during convalescence, resulting in only antegrade ureteral bleeding. Clip slippage is a well-known occurrence, especially during ligation of vessels (2). However, slippage from the ureter has never been reported. Alternatives to titanium clips include locking clips, staplers, suture, cautery, and various vessel-sealing systems. We now ligate the ureter with plastic locking clips during laparoscopic nephrectomies.

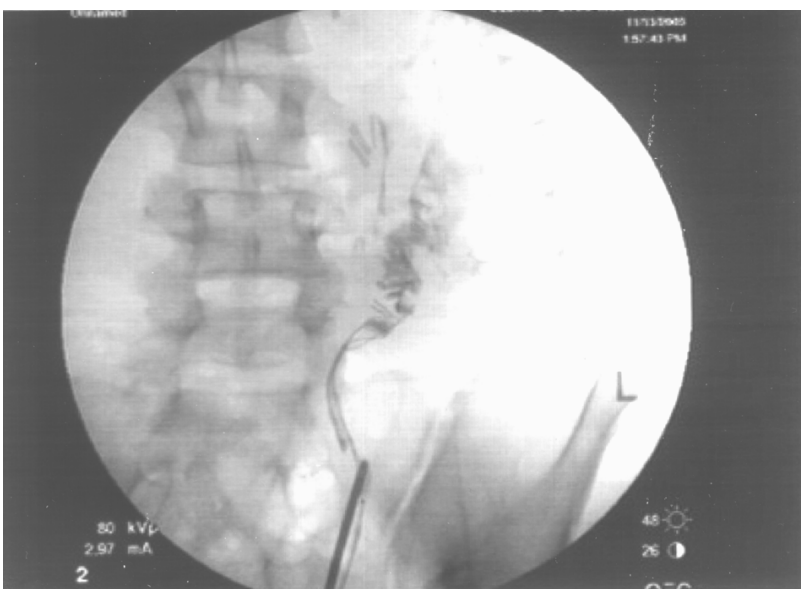

Figure 2 - Left retrograde injection of contrast medium demonstrating extravasation into the peritoneum.

In patients who present with delayed gross hematuria after any laparoscopic nephrectomy, bleeding from the ureteral stump should be considered. Failed conservative management should be followed by cystoscopic localization and, if ureteral-stump bleeding is identified, laparoscopic exploration. Imaging studies such as computed tomography and angiography might be helpful in demonstrating a fluid collection or active bleeding, respectively; however, findings may also be equivocal, thus delaying definitive treatment.

\section{REFERENCES}

1. Jacobs SC, Cho E, Foster C, Liao P, Bartlett ST: Laparoscopic donor nephrectomy: the University of Maryland 6-year experience. J Urol. 2004; 171: 47-51.

2. Carvi y Nievas MN, Hollerhage HG: Risk of intraoperative aneurysm clip slippage: a new experience with titanium clips. J Neurosurg. 2000; 92: 478-80.

Received: May 22, 2004

Accepted: June 30, 2004

\footnotetext{
Correspondence address:

Dr. Steve Y. Chung

Endourology Institute, Cedars-Sinai Medical Center

8635 West 3rd Street, Suite 1070

Los Angeles, California, 90048, USA

Fax: + $1310-423-4711$

E-mail: steve.chung@cshs.org
} 